\title{
HIGH-RESOLUTION FOURIER TRANSFORM SPECTROSCOPY WITH A VLT
}

\author{
Donald N.B. Hall \\ Space Telescope Science Institute, Baltimore, U.S.A.
}

The major advantages of the FTS technique are (1) multiplexing, (2)

throughput, (3) instrumental profile, (4) stability of frequency calibration, and (5) spectrophotometric accuracy. The multiplex advantage is realized only if one is detector noise limited for the signal within an individual spectral-resolution element. At optical and thermal infrared wavelengths, this is only the case at high spectral resolution $(\$ 50000)$ for modern detectors. By the time the VLT is operating one expects this to also be the case in the 1 - to 2.5 -micron region. At resolutions $>50000$ there are severe problems matching dispersive spectrographs to the VLT aperture, whereas existing FTS instruments already have adequate throughput to match to fields of a few arcsec with a VLT. When the other advantages are considered, the FTS is the instrument of choice for high-resolution ( $\geqslant 50000)$ spectroscopy of absorption features with a VLT. Foreseeable astrophysical applications include observations of interstellar and circumstellar features and of fully resolved profiles of photospheric and planetary lines.

The major disadvantage of the FTS is that it uses only a very small area of the VLT focal plane. If the incidence of extended fields or multiple objects suitable for high-resolution spectroscopy justifies it, fiber optic feeds or a field widened FIS design together with multiple or array detectors should be considered. Otherwise, the parallel use of some wide-field instrument in a serendipity mode would make full use of VLT observing time.

Proceedings of the IAU Colloquium No. 79: "Very Large Telescopes, their Instrumentation and Programs", Garching, April 9-12, 1984. 